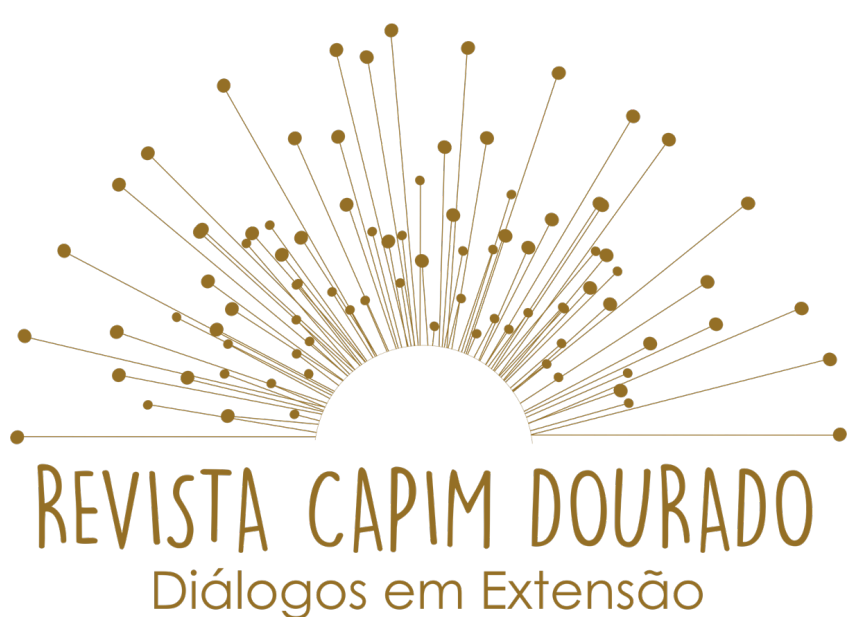

ISSN n² 2595-7341

Vol. 3, n. 2, Maio-Agosto, 2020

DOI: http://dx.doi.org/10.20873/uft.2595-7341.2020v3n2p199

\title{
APOIO EMOCIONAL A CUIDADORES DE IDOSOS: vivência de voluntários do projeto escuta solidária
}

EMOTIONAL SUPPORT FOR CARERS OF THE ELDERLY: experience of volunteers from the solidary listening project

APOYO EMOCIONAL PARA CUIDADORES DE MAYORES: experiencia de voluntarios del proyecto de escucha solidaria

Daniella Pires Nunes ${ }^{1}$, Jéssica da Silva Marinho², Ilaíse Brilhante Batista ${ }^{3}$,Yana Caroline Fernandes Ferreira4, Guiomar Virginia Vilela Assunção de Toledo Batello ${ }^{5}$, James Stefison Sousa Santos ${ }^{6}$, Bruna Mourão Moura ${ }^{7}$, Adriane Ribeiro da Silva Amend ${ }^{8}$, Fabiana Daronch ${ }^{9}$, Loan Aragão Piva ${ }^{10}$, Antônio Matheus Santos Medrado ${ }^{11}$, Taísa Souza Ribeiro ${ }^{12}$, Maria Sortênia Alves Guimarães $^{13}$,Andréia Queiroz Ribeiro ${ }^{14}$

1 Doutora em Enfermagem na Saúde do Adulto da Universidade de São Paulo, USP, Brasil, daniellanunes@unicamp.br.

2 Discente do curso de Licenciatura em Enfermagem da Universidade Federal do Tocantins, UFT, Brasil, jess.smarinho@gmail.com

3 Discente do curso de Enfermagem da Universidade Federal do Maranhão, UFMA, Brasil. IlaBrilhante@hotmail.com.

${ }^{4}$ Universidade Federal do Maranhão, UFMA, Brasil, yanacarolinef0@gmail.com.

${ }^{5}$ Mestra em Saúde Coletiva Universidade Luterana do Brasil, ULBRA, Brasil. guivilelatoledo@mail.uft.edu.br.

6 Discente do curso de Licenciatura em Enfermagem da Universidade Estadual Vale do Acaraú, UVA-CE, Brasil, jamesstefison@gmail.com

${ }^{7}$ Mestra em Ciências e Saúde da Universidade Federal do Tocantins, UFT, Brasil, brunavras@hotmail.com.

8 Discente do curso de Licenciatura em Enfermagem Universidade Federal do Tocantins, UFT, Brasil, adrianeamend@hotmail.com.

9 Mestre em Ciências da Saúde da Universidade Federal do Tocantins, UFT, Brasil, fabianadaronch@uft.edu.br.

10 Discente do curso de Licenciatura em Enfermagem da Universidade Federal do Tocantins, UFT, Brasil, loantuc123@gmail.com.

11 Discente do curso de Enfermagem da Universidade Federal do Tocantins, UFT, Brasil, mmedrado7@gmail.com.

12 Discente do curso de Licenciatura em Enfermagem da Universidade Estadual de Feira de Santana, UEFS, Brasil, taisaribeiro08@gmail.com.

13 Doutora em CIÊNCIA DA NUTRIÇÃO - PPGCN da Universidade Federal de Viçosa, UFV, Brasil, msortenia@uft.edu.br.

${ }^{14}$ Discente do curso de Licenciatura em odontologia da Universidade Federal de Juiz de Fora, UFJF, Brasil, andreia.ribeiro@ufv.br. 


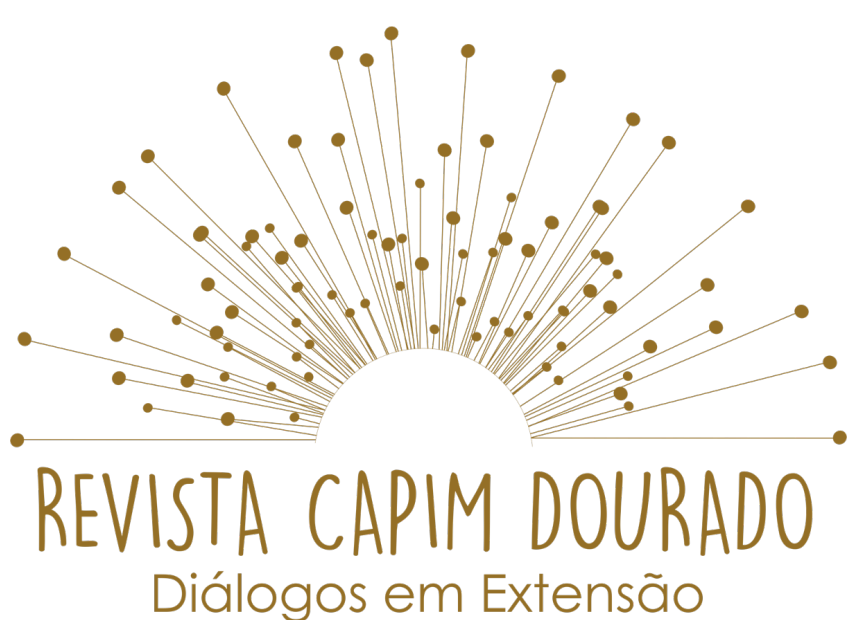

ISSN n² 2595-7341

Vol. 3, n. 2, Maio-Agosto, 2020

DOI: http://dx.doi.org/10.20873/uft.2595-7341.2020v3n2p199

\section{RESUMO}

Esse trabalho objetivou relatar a vivência de voluntários em ações do Projeto Escuta Solidária voltadas aos cuidadores de idosos acamados. Os voluntários realizavam ligações telefônicas que possibilitaram o estreitamento de vínculo com cuidadores, permitindo o desenvolvimento de habilidades como 0 acolhimento, a escuta qualificada e o reconhecimento das necessidades do cuidador. A experiência perpassou o conhecimento científico, promovendo aos voluntários percepção de gratidão e engrandecimento na execução das ações.

PALAVRAS-CHAVE: Cuidadores; Idoso fragilizado; Apoio Social; Pandemia.

\section{ABSTRACT}

This work aimed to report the experience of volunteers in actions of the Escuta Solidária Project aimed at caregivers of bedridden elderly. The volunteers made telephone calls that made it possible to strengthen the bond with caregivers, allowing the development of skills such as reception, qualified listening and recognition of the caregiver's needs. The experience permeated scientific knowledge, promoting volunteers' perception of gratitude and aggrandizement in the execution of actions.

KEYWORDS: Caregivers; Frail Elderly; Social Support; Pandemics.

\section{RESUMEN}

Este trabajo tuvo como objetivo informar la experiencia de los voluntarios en las acciones del Proyecto Escuta Solidária dirigido a los cuidadores de ancianos en cama. Los voluntarios hicieron llamadas telefónicas que permitieron fortalecer el vínculo con los cuidadores, permitiendo el desarrollo de habilidades tales como la recepción, la escucha calificada y el reconocimiento de las necesidades del cuidador. La experiencia impregna el conocimiento científico, promoviendo la 


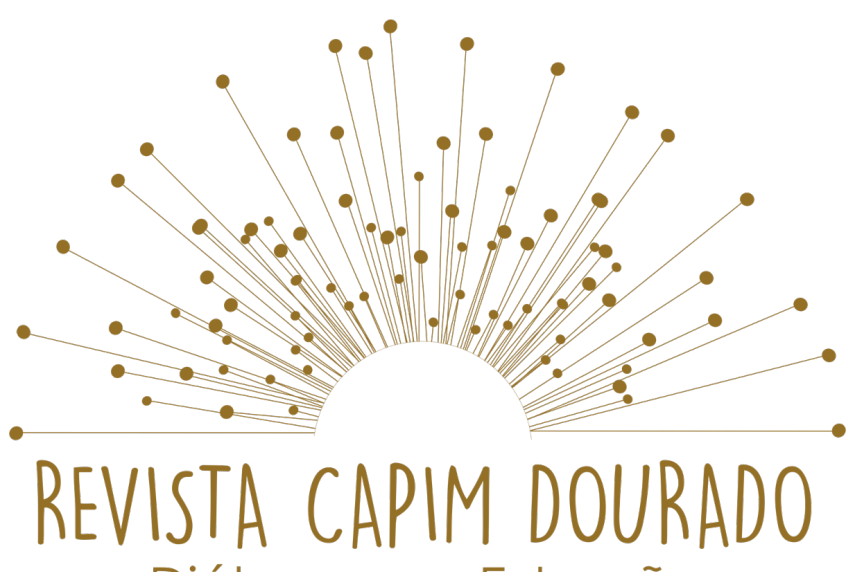

Diálogos em Extensão

ISSN n 2595-7341 Vol. 3, n. 2, Maio-Agosto, 2020

DOI: http://dx.doi.org/10.20873/uft.2595-7341.2020v3n2p199

percepción de gratitud y engrandecimiento de los voluntarios en la ejecución de las acciones.

PALABRAS CLAVE: Cuidadores; Anciano Frágil; Apoyo Social; Pandemia.

Recebido em: 31.03.2020. Aceito em: 19.04.2020. Publicado em: 30.05.2020. 


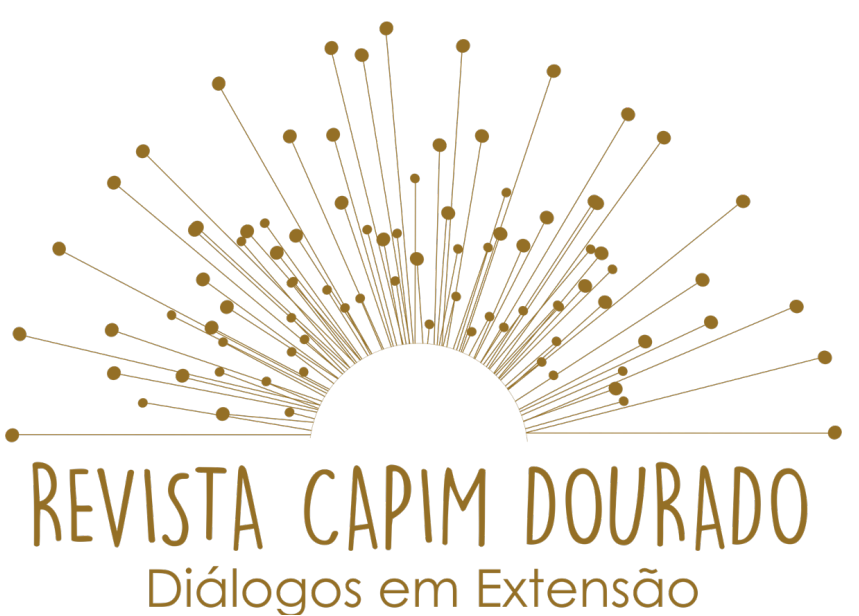

ISSN n² 2595-7341

Vol. 3, n. 2, Maio-Agosto, 2020

DOI: http://dx.doi.org/10.20873/uft.2595-7341.2020v3n2p199

\section{O cuidador de idosos no contexto da pandemia de COVID-19}

A pandemia de Coronavírus Disease 2019 (COVID-19), decretada em março de 2020, possui elevada infectividade e acomete toda a população, sobretudo grupos específicos considerados de risco como idosos e indivíduos com doenças crônicas (ANDERSON et al, 2020).

A pessoa idosa, além do risco para COVID-19, pode apresentar condições inerentes ao envelhecimento que a predispõe à incapacidade funcional e, consequentemente, exigirá a presença de um cuidador. O cuidador é a pessoa que assume a responsabilidade dos cuidados básicos de maneira esporádica ou em tempo integral, e pode ser ou não familiar (NUNES et al., 2018).

Em situações de crises, como na pandemia, os cuidados são redobrados em virtude da adoção de medidas preventivas para conter a propagação do vírus além das demandas diárias, no intuito de garantir a segurança do idoso (GARCIA; DUARTE, 2020; ANDERSON et al, 2020). Esse fato pode contribuir para o surgimento de medo, anseio, preocupação e tristeza, o que pode piorar a saúde mental, emocional e física do cuidador.

Diante desse cenário, o cuidador necessita de uma rede de apoio que amenize os impactos negativos do cuidar neste cenário de crise (KOBAYASI et al, 2019). Considera-se rede de apoio um grupo de pessoas que mantém contato ou vínculo com o indivíduo, que oferece informações, auxílio material, emocional ou afetivo. $\mathrm{O}$ apoio emocional relaciona-se ao recebimento de demonstrações de afeto positivo e de empatia; e é encorajado a expressar sentimentos de confiança, carinho, estima, escuta e interesse (BOWLING, 1997). 


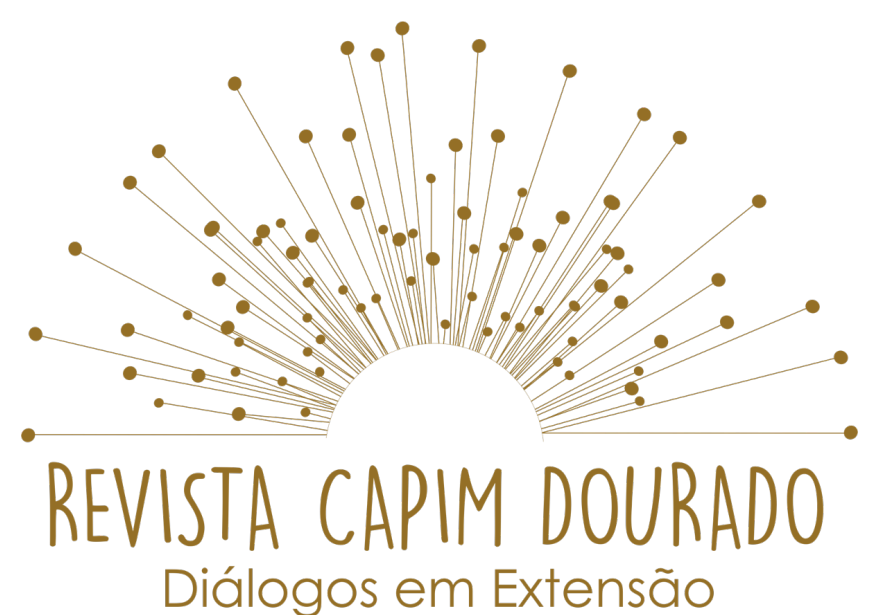

ISSN n² 2595-7341

Vol. 3, n. 2, Maio-Agosto, 2020

DOI: http://dx.doi.org/10.20873/uft.2595-7341.2020v3n2p199

Com intuito de estabelecer estratégias de enfrentamento em tempos de pandemia, o Projeto Escuta Solidária visa o acolhimento e apoio emocional aos cuidadores. E integra a linha de extensão Saúde Humana, uma vez que prima pela promoção da saúde física e emocional e na melhoria da qualidade de vida e bemestar dos cuidadores de idosos.

Dessa forma o objetivo deste trabalho foi relatar a vivência de voluntários em ações do Projeto Escuta Solidária voltadas aos cuidadores de idosos acamados, durante a pandemia.

\section{Percurso metodológico}

Trata-se de um relato de experiência vivenciado por voluntários (discentes da graduação e pós-graduação) participantes do Projeto Escuta Solidária (PES), no município de Palmas (TO), entre os meses de abril a junho de 2020.

O PES é uma iniciativa de docentes das Universidade Federal de Viçosa (UFV), Federal do Tocantins (UFT) e Estadual de Campinas (Unicamp) com intuito de realizar a escuta qualificada à pessoa idosa durante o distanciamento social, amenizar sentimento de solidão e reforçar orientações acerca das medidas de proteção individual contra o coronavírus.

A partir dessa proposta, a líder do Grupo de Pesquisa Envelhecimento e Cuidado (GPEC) da UFT estabeleceu parceria com a coordenadora do PES-Palmas para que esta ação fosse ampliada aos cuidadores de idosos. Membros do GPEC tem avaliado as condições de vida e saúde dos cuidadores de idosos acamados neste município, e no auge da pandemia as atividades foram suspensas. 


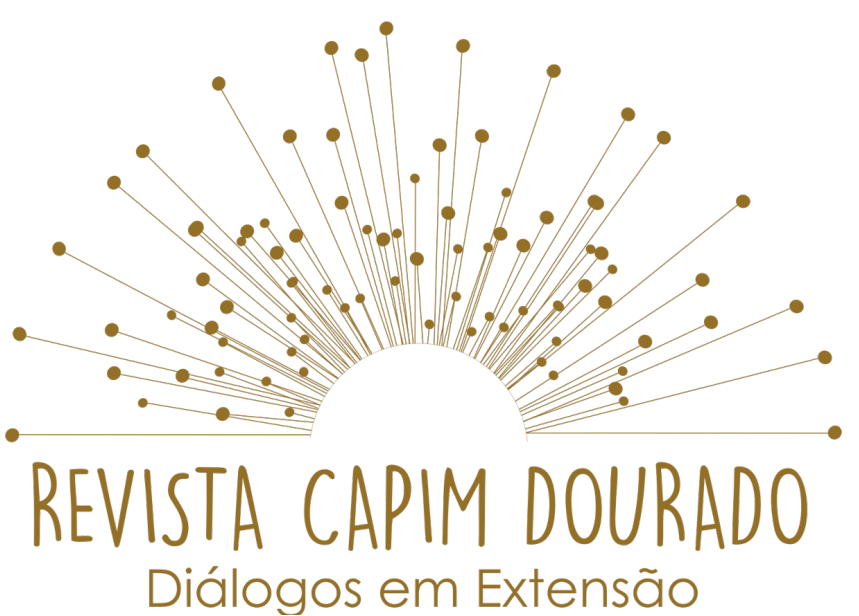

ISSN n² 2595-7341

Vol. 3, n. 2, Maio-Agosto, 2020

DOI: http://dx.doi.org/10.20873/uft.2595-7341.2020v3n2p199

Para a execução das ações da escuta qualificada realizaram-se as seguintes etapas:

a) Orientação dos voluntários: dez membros do GPEC participaram do projeto e foram orientados sobre o intuito do PES e as ações a serem executadas. Disponibilizou-se para os voluntários um manual com as orientações sobre a conduta durante as ligações bem como de orientações sobre medidas de prevenção da COVID-19.

b) Recrutamento dos cuidadores: o grupo de pesquisa possui lista de idosos acamados e seus respectivos cuidadores, que foi disponibilizada pela Secretaria de Saúde de Palmas. A seleção dos cuidadores ocorreu de forma aleatória, e estabeleceu-se como critério de exclusão, os cuidadores que não foram contatados a partir de três tentativas de contato (via telefone ou WhatsApp).

c) Realização e acompanhamento da Escuta Solidária: os voluntários contatavam os cuidadores por ligação telefônica ou WhatsApp, e explicitava o objetivo do projeto e caso aceitasse participar, iniciava-se a escuta qualificada. Ademais, durante a escuta, os voluntários preenchiam um formulário de acompanhamento com questões sobre a escuta e de condições física e emocional do indivíduo.

Com intuito de ofertar apoio emocional durante as escutas, os voluntários estabeleciam com o cuidador a frequência de acompanhamento que poderia ser semanal ou quinzenal, de acordo com a necessidade e disponibilidade dele.

Tecendo apoio aos cuidadores de idosos em tempos de pandemia. 


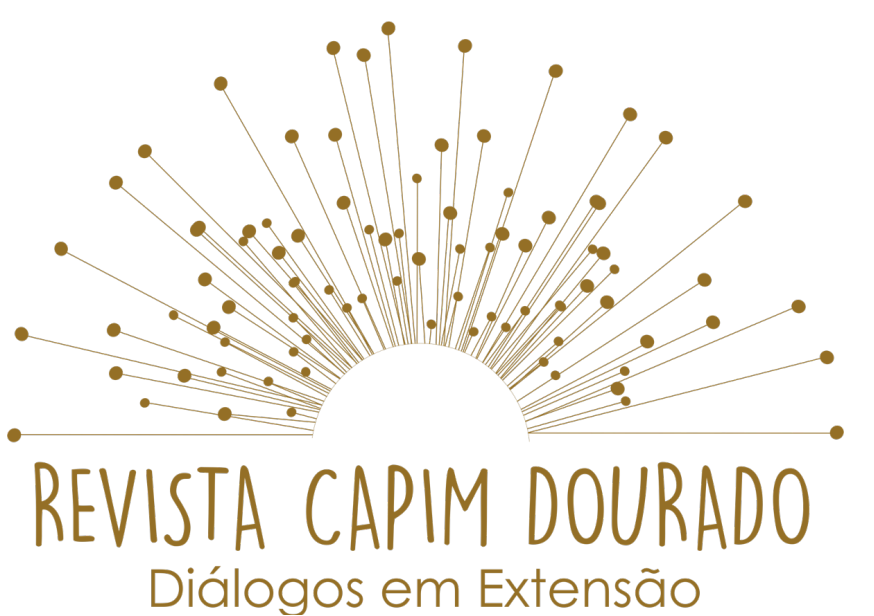

ISSN n² 2595-7341

Vol. 3, n. 2, Maio-Agosto, 2020

DOI: http://dx.doi.org/10.20873/uft.2595-7341.2020v3n2p199

Foram acompanhados 32 cuidadores, sendo a maioria mulher, familiar, com médias de idade de 48 anos e de cuidado, 5,6 anos. No primeiro contato, os cuidadores demonstraram-se receptivos e o diálogo incluía perguntas simples para que falasse sobre as atividades realizadas para si e das relativas ao cuidado do idoso, e também sobre a medidas preventivas da COVID-19, como o uso de máscaras ao sair do domicílio, importância da higienização das mãos, lavagem dos alimentos que são comprados no supermercado/feira e cuidados gerais com cuidados gerais com a higienização e/ou limpeza da casa.

A criação de vínculo foi gradual a partir das ligações subsequentes. Tal fato justifica-se pelo medo e receio do estabelecimento de diálogo com desconhecidos. As ligações subsequentes permitiram que o vínculo fosse estreitado e possibilitou que os cuidadores sentissem mais confortáveis na partilha sobre suas narrativas de histórias de vida, emoções, sentimentos e cotidiano.

As principais queixas relatadas por eles, durante a pandemia, foram: medo, preocupação, ansiedade, estresse, solidão, cansaço pelas demandas intensas de cuidado, falta de ajuda de outras pessoas para auxiliar no cuidado ao idoso. Notaram-se que os cuidadores priorizam e destinam a maior parte do seu tempo para o cuidado, colocando suas necessidades em segundo plano. Dessa forma, os cuidadores também necessitam de um olhar mais individualizado, com cuidados e apoio, para que promovam uma melhor qualidade de vida.

A partir do reconhecimento das necessidades dos cuidadores, os voluntários implementavam ações estratégicas para provimento de possíveis soluções. Os cuidadores foram orientados sobre a necessidade de organizar um 


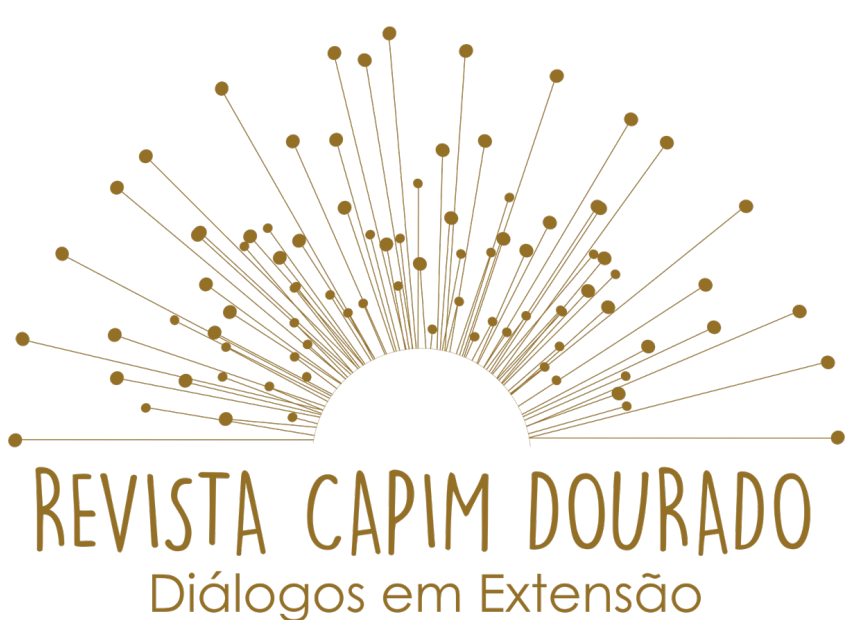

ISSN n² 2595-7341

Vol. 3, n. 2, Maio-Agosto, 2020

DOI: http://dx.doi.org/10.20873/uft.2595-7341.2020v3n2p199

tempo para seu autocuidado como a prática de exercícios físicos, leitura de um livro, realizar trabalhos manuais como crochê, assistir um filme/novelas, cuidar do jardim, entre outros. Ressalta-se que as atividades foram sugeridas de acordo com a singularidade de cada cuidador e que the trouxesse satisfação, promovendo assim a valorização de si mesmo.

Também foram solicitadas pelos cuidadores demandas relativas ao cuidado com o idoso como a solicitação de fraldas, cadeiras de rodas e direcionados aos serviços de saúde e serviços sociais, dentre elas a participação da campanha de vacinação e o requerimento de cestas básicas.

Algumas situações dificultaram a realização da ação como os contatos telefônicos desatualizados e dificuldade no agendamento de horário entre voluntário e cuidador. No entanto, a avaliação das ações do projeto foi benéfica para o cuidador tendo em vista a receptividade emanada por eles.

Dentre as falas dos cuidadores, destacam-se os relatos de terem sido "lembrados"e "vistos", neste momento de pandemia, pois a atenção geralmente é para o idoso. O projeto preocupou-se com as demandas desses atores sociais e permitiu um olhar singular para o provedor de cuidado, pois se ele está bem, ele cuida bem.

O projeto possibilitou aos voluntários o estabelecimento de vínculo entre universidade e comunidade, permitiu o reconhecimento da tríade do cuidado profissional-cuidador-idoso por meio de uma visão ampliada da linha do cuidado integral que perpassa pelo vínculo, responsabilização e acolhimento, mediante o uso das tecnologias leves (as tecnologias das relações) para o cuidado. Nessa 


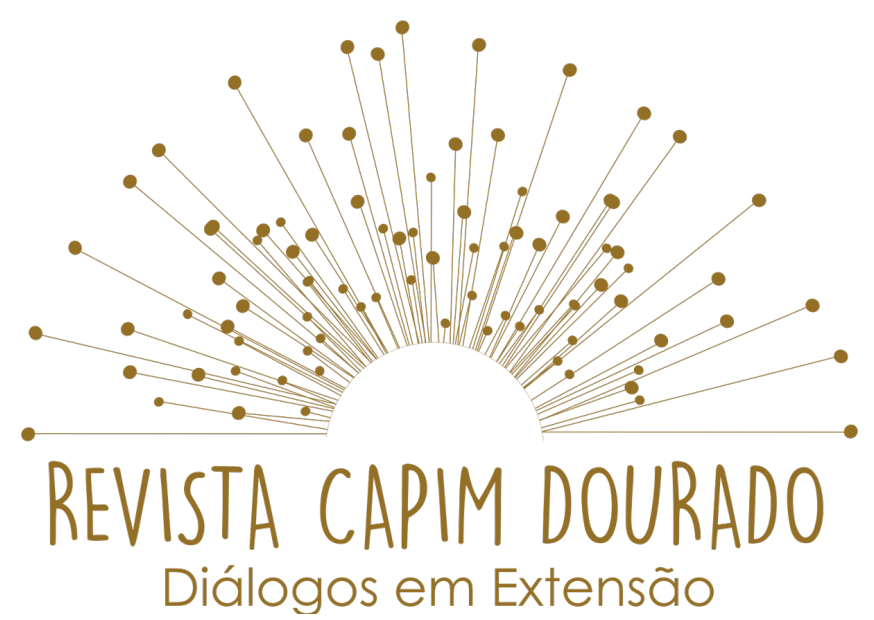

ISSN n² 2595-7341

Vol. 3, n. 2, Maio-Agosto, 2020

DOI: http://dx.doi.org/10.20873/uft.2595-7341.2020v3n2p199

perspectiva, as habilidades como o acolhimento, a escuta qualificada e o reconhecimento das necessidades do cuidador foram desenvolvidas.

Dessa forma, a ação de promoção de saúde realizada por meio da escuta qualificada, perpassou o conhecimento científico, promovendo aos voluntários uma experiência de vivência significativa, tendo em vista, a percepção de gratidão e engrandecimento na execução das ações.

Após o encerramento do distanciamento social pelas instituições de saúde, os cuidadores serão contatados para continuidade das ações de pesquisa desenvolvidas pelo GPEC.

\section{CONSIDERAÇÕES FINAIS}

Os voluntários do PES relataram que a vivência com cuidadores de idosos foi enriquecedora por demonstrar a importância de integração do ensino teórico à prática, o desenvolvimento de vínculo e um olhar crítico para as condições dos envolvidos. Ressaltam-se a relevância das ações extensionistas no meio universitário por ser um elo entre os futuros profissionais à comunidade.

\section{REFERÊNCIAS}

ANDERSON, R. M. et al. How will country-based mitigation measures influence the course of the COVID-19 epidemic? The Lancet. v. 395, n. 10228. 2020. DOI: https://doi.org/10.1016/S0140-6736(20)30567-5.

BOWLING, A. Measuring social networks and social support. In: Measuring Health: a Review of Quality of Life Measurements Scales. Baltimore: Open University Press, 1997. p. 91-109. 


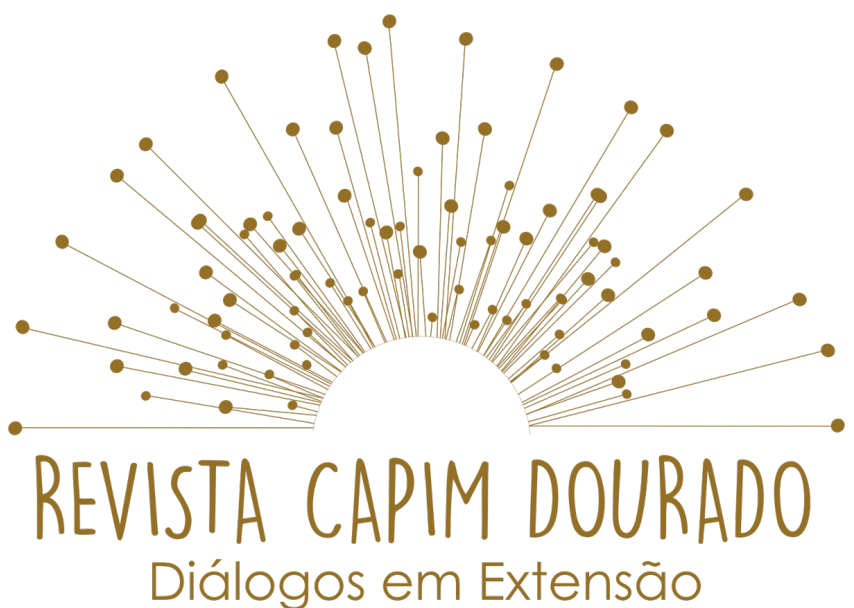

ISSN n² 2595-7341

Vol. 3, n. 2, Maio-Agosto, 2020

DOI: http://dx.doi.org/10.20873/uft.2595-7341.2020v3n2p199

GARCIA, L. P.; DUARTE, E. Intervenções não farmacológicas para o enfrentamento à epidemia da COVID-19 no Brasil. Epidemiol. Serv. Saude. v. 29, n. 2, 2020. DOI: https://doi.org/10.5123/S1679-49742020000200009.

KOBAYASI, D. Y. et al. Sobrecarga, rede de apoio social e estresse emocional do cuidador do idoso. av.enferm. Bogotá, v. 37, n. 2, p. 140-148, ago, 2019. DOI: http://dx.doi.org/10.15446/av.enferm.v37n2.73044.

NUNES, D. P. et al. Cuidadores de idosos e tensão excessiva associada ao cuidado: evidências do Estudo SABE. Rev. bras. epidemiol. São Paulo, v. 21, supl. 2, e.180020, 2018. DOI: https://doi.org/10.1590/1980-549720180020.supl.2. 